\title{
ON THE CHOICE OF SHOCK CAPTURING SCHEMES FOR THE SOLUTION OF THE LWR TRAFFIC FLOW EQUATION USING A HIGH ORDER MODAL DISCONTINUOUS GALERKIN DISCRETIZATION
}

\author{
Alberto Costa Nogueira Junior ${ }^{1}$, João Lucas de Sousa Almeida ${ }^{1}$, Cláudio Alessandro de \\ Carvalho Silva ${ }^{2}$ \\ ${ }^{1}$ IBM Research Brazil \\ Rua Tutóia, 1157 - São Paulo/SP - 04007-900 \\ e-mail: \{albercn, jlsa\}@br.ibm.com \\ 2 Thorus Scisoft Tecnologia \\ Rua Francisco Glicério, 1561 - Valinhos/SP - 13271-200 \\ e-mail: cacs@thorus-scisoft.com.br
}

Keywords: Traffic Flow, LWR Equation, High-Order, Modal, Discontinuous Galerkin, Shock Capturing.

\begin{abstract}
In this paper we studied different techniques to stabilize the numerical solution of the LWR traffic flow equation when using a modal version of the high order discontinuous Galerkin finite element method (DGFEM). Based on a set of three standard examples of traffic flow initial conditions, we compared four shock capturing strategies to control Gibbs phenomenon around discontinuous solutions of the car density conserved variable: (1) generalized slope limiter $\left(\Pi^{N}\right)$, (2) hierarchical slope limiter, (3) sub-cell shock capturing with elementwise constant artificial viscosity, and (4) sub-cell shock capturing with local $C^{0}$ artificial viscosity. Although such stabilizing techniques were well documented for aerospace applications, our experiments revealed important features which are worth mentioning. Firstly, regardless of the polynomial order of the approximation, both limiters required quite refined meshes to achieve sharp shock resolution. Secondly, shock capturing based on elementwise constant viscosity produced small traveling spikes over the solution profiles destroying the locality of the method and resulting in excessive smearing of the discontinuities. Finally, $C^{0}$ artificial viscosity addition produced sharp shock resolutions with very coarse meshes and high order polynomial approximations. Overall, this technique demonstrated the better cost-benefit in terms of computational effort (number of degrees of freedom) to achieve a certain level of shock resolution.
\end{abstract}




\section{INTRODUCTION}

The LWR nonlinear equation due to Lighthill, Witham and Richards $[1,2,3]$ is one of the most popular PDEs used to describe traffic flow problems. The model dynamics is written as a scalar hyperbolic conservation law in one dimension [4]. The LWR mathematical formulation assumes a monotonically decreasing relation between speed and density of cars leading to a strictly concave form of the convective flux associated to the hyperbolic PDE [5].

In this paper we discretized the LWR equation using the modal version of the high order discontinuous Galerkin method (DGM) [6]. The Lax-Friedrichs' numerical flux was chosen to approximate the solution of Riemman problems at element boundaries within the mesh. Details of the DGM numerical formulation implemented in this work can be found in [7]. As the unsteady LWR scalar equation naturally develops shocks along time integration and we wanted to use high order approximations, we were specially interested in shock capturing strategies to stabilize solutions and get rid of undesirable wiggles.

The main goal of the present study is to implement and compare different shock capturing strategies to stabilize the numerical solution of the LWR equation and establish the most suited way to deal with Gibbs phenomenon in terms of shock resolution and computational efficiency. Four well known stabilizing techniques were considered to accomplish this task: (1) generalized slope limiter $\left(\Pi^{N}\right)$ [8]; (2) hierarchical slope limiter [9]; (3) sub-cell shock capturing with elementwise constant artificial viscosity [11] and (4) sub-cell shock capturing with local $C^{0}$ artificial viscosity [12]. We used the LDG numerical flux [10, 8] to discretize the $2^{\text {nd }}$ order diffusive operator associated to the viscous formulation of the LWR equation when considering the stabilization techniques based on artificial viscosity. A five step $4^{\text {th }}$ order strong stability preserving Runge-Kutta explicit integrator [8] was used to evolve the semi-discrete formulation in time. Numerical examples for typical traffic flow situations were chosen to validate and compare the algorithmic performance. The following examples, with three different initial conditions, were evaluated: (a) red light at road exit; (b) green light at half way of road length and (c) exponential car density at road entrance.

This paper is organized as follows: section 2 introduces the LWR equation and its corresponding boundary value problem, section 3 briefly describes the DGFEM discretization used to numerically approximate the solution of the LWR boundary value problem, section 4 discusses the four different shock capturing strategies considered to stabilize the numerical solutions, section 5 discusses the results obtained when comparing all shock capturing mechanisms through a set of three typical examples of traffic flow initial conditions, finally, section 6 summarizes the main results and point out the strongest and weakest features of each shock capturing scheme used in our numerical experiments.

\section{THE LWR TRAFFIC FLOW MODEL}

To model traffic flow behavior along single roads the present study considers the LighthillWhitham-Richards (LWR) equation which can be written as follows,

$$
\frac{\partial u(x, t)}{\partial t}+\frac{\partial f(u(x, t))}{\partial x}=0, x \in[L, R]=\Omega,
$$

with appropriate initial and boundary conditions given by

$$
u(x, 0)=u_{0}(x), u(L, t)=u_{L}(t), u(R, t)=u_{R}(t),
$$

where $u(x, t)$ is the traffic density, $x \in \mathbb{R}$ and $t \in \mathbb{R}_{+}$are the space and time coordinates and $\Omega$ is the problem domain, respectively. 
The main conserved quantity in the LWR model is the traffic density which is defined as the average number of cars per unit length of road. A fundamental assumption of this model is the car speed as a monotonically decreasing function of the car density. Such hypothesis, postulated in [5], implies the following quadratic flux function $f: \mathbb{R} \rightarrow \mathbb{R}$ in equation 1 ,

$$
f(u)=V_{\max }\left(u-\frac{u^{2}}{u_{\max }}\right)
$$

where the constants $V_{\max }$ and $u_{\max }$ stand for maximum car speed and maximum car density, respectively.

Fig. 1 depicts the monotonically decreasing car speed as a function of car density as well as the resulting quadratic flux described in equation 3.
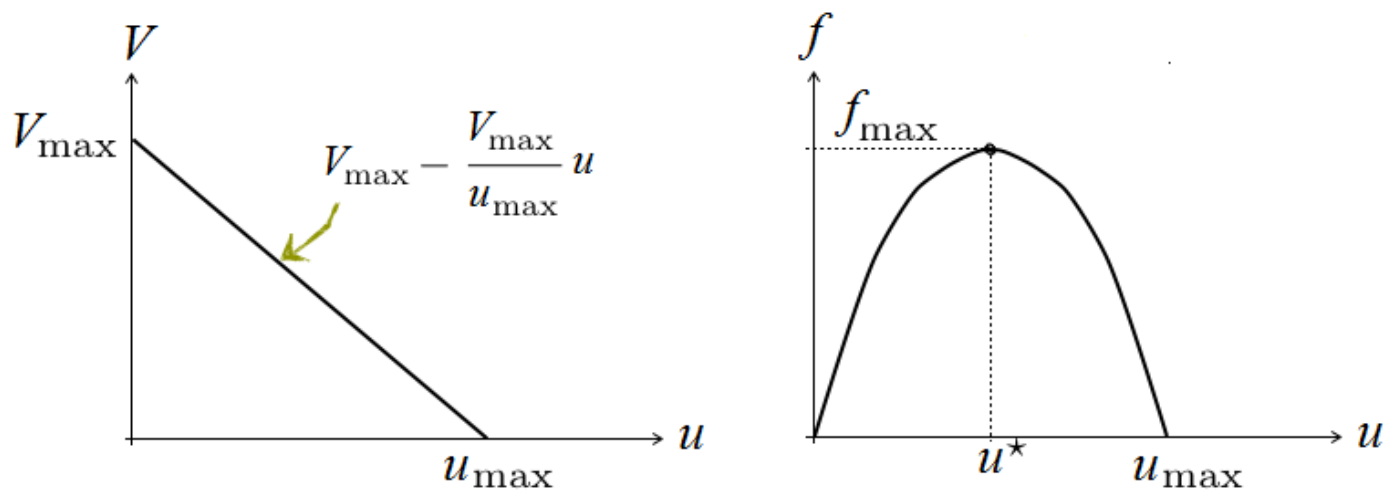

Figure 1: Monotonically decreasing car speed and quadratic traffic flux.

\section{DGFEM DISCRETIZATION}

Following a standard DGFEM approach, we rewrite the boundary value problem defined by equations 1 and 2 in a weak form valid for each grid element separately, and enforce a global approximate solution choosing a numerical flux at each element boundary to establish communication among elements.

We consider an approximation of $\Omega$ by $K$ non-overlapping elements, $x \in\left[x_{l}^{k}, x_{r}^{k}\right]=\Omega^{k}$ and for each grid element $\Omega^{k}$, we start forming the local residual as follows

$$
x \in \Omega^{k}: \mathcal{R}_{h}(x, t)=\frac{\partial u_{h}^{k}}{\partial t}+\frac{\partial f_{h}^{k}}{\partial x},
$$

where $u_{h}^{k}$ and $f_{h}^{k}$ are numerical approximations to the traffic density, $u(x, t)$, and the physical flux, $f(u)$, respectively. We also assume that both functions can be expressed as polynomials of order $\mathrm{N}$

$$
\begin{aligned}
x \in \Omega^{k}: u_{h}^{k}(x, t) & =\sum_{j=1}^{N+1} \hat{u}_{j}^{k}(t) \psi_{j}^{k}(x), \\
f_{h}^{k}\left(u_{h}(x, t)\right) & =\sum_{j=1}^{N+1} \hat{f}_{j}^{k}(t) \psi_{j}^{k}(x) .
\end{aligned}
$$


where $\psi_{j}^{k}(x)$ are polynomial basis functions belonging to a finite dimensional vector space $V_{h}^{k}=\operatorname{span}\left\{\psi_{j}^{k}\left(\Omega^{k}\right)\right\}_{j=1}^{N+1}$. In this work, we considered the Legendre polynomials, $P_{n}(\xi)$, $\xi \in[-1,1]$, as our set of modal basis functions defined over the reference element $\hat{\Omega}$, i.e., $\psi_{j}^{k}(x(\xi))=P_{n}(\xi)$.

Returning to equation 4 , we require the residual to be orthogonal to every basis function $\psi_{j}^{k}(x)$ belonging to the vector space $V_{h}^{k}$,

$$
\int_{\Omega^{k}} \mathcal{R}_{h}(x, t) \psi_{i}^{k}(x) d x=0, \quad 1 \leq i \leq N+1
$$

After integrating by parts the expression 7, we arrive at the local weak form of the LWR equation

$$
\int_{\Omega^{k}}\left(\frac{\partial u_{h}^{k}}{\partial t} \psi_{i}^{k}(x)-f_{h}^{k}\left(u_{h}^{k}\right) \frac{d \psi_{i}^{k}(x)}{d x}\right) d x=-\left[\psi_{i}^{k}(x) f^{*}\right]_{x_{l}^{k}}^{x_{k}^{k}}, 1 \leq i \leq N+1 .
$$

At this point it is necessary to define the numerical flux $f^{*}$. We chose the Lax-Friedrichs flux because of its simplicity and effectiveness.

After some tedious algebra manipulation, we can write the matrix form of our local semidiscrete weak statement [7]

$$
\begin{aligned}
\frac{h^{k}}{2} \mathcal{M} \frac{d}{d t} \hat{\boldsymbol{u}}_{h}^{k}-\mathcal{S}^{T} \hat{\boldsymbol{f}}_{h}^{k}=[ & -\frac{1}{2} \mathcal{F}_{l}^{k+1}\left(\hat{\boldsymbol{f}}_{h}^{k+1}-C \hat{\boldsymbol{u}}_{h}^{k+1}\right)-\frac{1}{2} \mathcal{F}_{r}^{k}\left(\hat{\boldsymbol{f}}_{h}^{k}+C \hat{\boldsymbol{u}}_{h}^{k}\right) \\
& \left.+\frac{1}{2} \mathcal{F}_{r}^{k-1}\left(\hat{\boldsymbol{f}}_{h}^{k-1}+C \hat{\boldsymbol{u}}_{h}^{k-1}\right)+\frac{1}{2} \mathcal{F}_{l}^{k}\left(\hat{\boldsymbol{f}}_{h}^{k}-C \hat{\boldsymbol{u}}_{h}^{k}\right)\right],
\end{aligned}
$$

where $\mathcal{M}$ and $\mathcal{S}$ are the local mass and stiffness matrices, respectively. The term $h^{k} / 2$ is the jacobian associated to the affine transformation between the reference element $\hat{\Omega}=[-1,1]$ and each grid element $k$-th. In the above formula we also identify the Lift matrices, $\mathcal{F}$, associated to the boundaries of each element. These matrices are defined as the dyadic products of elemental basis functions evaluated at the boundaries of the standard element $\hat{\Omega}$ [7]. The vector variables $\hat{\boldsymbol{u}}_{h}^{k}$ and $\hat{\boldsymbol{f}}_{h}^{k}$ account for the degrees of freedom of the model and their meaning were already described by expressions 5 and 6 . It is worth mentioning that we locally recover $\hat{f}_{i}^{k}$ coefficients, in terms of the approximate solution $u_{h}^{k}$, through a $L^{2}$ projection as detailed in [7]. Finally, the variable $C$ is defined as the upper bound of the characteristic wave speed of the approximate numerical solution.

When dealing with artificial viscosity based techniques for shock capturing, we must write a viscous version of the original LWR equation. Thus, we add a second order diffusive term at the right hand side of equation 1 and define an auxiliary variable $q$ to keep the DG approach of the numerical approximation which contains only first order derivatives. The resulting system of first order equations is now given as,

$$
\begin{aligned}
& \frac{\partial u}{\partial t}+\frac{\partial}{\partial x}(f(u)-\sqrt{\varepsilon} q)=0 \\
& q=\sqrt{\varepsilon} \frac{\partial u}{\partial x}
\end{aligned}
$$

where $\varepsilon(x)$ is the artificial viscosity function. 
As before, we assume that the auxiliary variable $q$ which approximates the derivative of the conserved variable $u(x, t)$ can be written as a polynomial expansion of order $N$ as follows,

$$
q_{h}^{k}(x, t)=\sum_{j=1}^{N+1} \hat{q}_{j}^{k}(t) \psi_{j}^{k}(x) .
$$

Following the same discretization steps applied to the original equation we arrive at the local weak statement of the modified traffic flow problem,

$$
\begin{aligned}
& \int_{\Omega^{k}} \frac{\partial u_{h}^{k}}{d t} \psi_{i}^{k}(x) d x-\int_{\Omega^{k}} f_{h}^{k}\left(u_{h}^{k}\right) \frac{d \psi_{i}^{k}(x)}{d x} d x+\int_{\Omega^{k}} \sqrt{\varepsilon} q_{h}^{k}\left(u_{h}^{k}\right) \frac{d \psi_{i}^{k}(x)}{d x} d x=-\left[\psi_{i}^{k}(x) f^{*}\right]_{x_{l}^{k}}^{x_{r}^{k}} \\
& +\left[\psi_{i}^{k}(x)\left(\sqrt{\varepsilon} q_{h}^{k}\right)^{*}\right]_{x_{l}^{k}}^{x_{r}^{k}}, 1 \leq i \leq N+1 \\
& \int_{\Omega^{k}} q_{h}^{k}\left(u_{h}^{k}\right) \psi_{i}^{k}(x) d x+\int_{\Omega^{k}} u_{h}^{k} \frac{d}{d x}\left(\sqrt{\varepsilon} \psi_{i}^{k}(x)\right) d x=\left[\psi_{i}^{k}(x)\left(\sqrt{\varepsilon} u_{h}^{k}\right)^{*}\right]_{x_{l}^{k}}^{x_{r}^{k}}, 1 \leq i \leq N+1 .
\end{aligned}
$$

The numerical fluxes $(\sqrt{\varepsilon} q)^{*}$ and $(\sqrt{\varepsilon} u)^{*}$ above, corresponding to the second order dissipative operator, were approximated by the Local Discontinuous Galerkin (LDG) flux which shows optimal convergence rates [10].

After choosing Lax-Friedrichs and LDG as our numerical fluxes and upon substitution of the polynomial expansions for $u_{h}^{k}, f_{h}^{k}, q_{h}^{k}$ in equations 12 and 13, we can write the final matrix form of the semi-discrete DGFEM approximation of the viscous version of LWR traffic flow equation,

$$
\begin{aligned}
\frac{h^{k}}{2} \mathcal{M} \frac{d}{d t} \hat{\boldsymbol{u}}_{h}^{k}-\mathcal{S}^{T} \hat{\boldsymbol{f}}_{h}^{k}+\left(\mathcal{S}^{\sqrt{\varepsilon}}\right)^{T} \hat{\boldsymbol{q}}_{h}^{k}= & -\frac{1}{2} \mathcal{F}_{l}^{k+1}\left(\hat{\boldsymbol{f}}_{h}^{k+1}-C \hat{\boldsymbol{u}}_{h}^{k+1}\right)-\frac{1}{2} \mathcal{F}_{r}^{k}\left(\hat{\boldsymbol{f}}_{h}^{k}+C \hat{\boldsymbol{u}}_{h}^{k}\right) \\
& +\frac{1}{2} \mathcal{F}_{r}^{k-1}\left(\hat{\boldsymbol{f}}_{h}^{k-1}+C \hat{\boldsymbol{u}}_{h}^{k-1}\right)+\frac{1}{2} \mathcal{F}_{l}^{k}\left(\hat{\boldsymbol{f}}_{h}^{k}-C \hat{\boldsymbol{u}}_{h}^{k}\right) \\
& \left.+\sqrt{\varepsilon\left(x_{r}^{k}\right)} \mathcal{F}_{r}^{k} \hat{\boldsymbol{q}}_{h}^{k}-\sqrt{\varepsilon\left(x_{r}^{k-1}\right)} \mathcal{F}_{r}^{k-1} \hat{\boldsymbol{q}}_{h}^{k-1}\right], \\
\frac{h^{k}}{2} \mathcal{M} \hat{\boldsymbol{q}}_{h}^{k}+\left(\tilde{\mathcal{S}}^{\sqrt{\varepsilon}}\right)^{T} \hat{\boldsymbol{u}}_{h}^{k}= & +\frac{1}{2}\left(\sqrt{\varepsilon\left(x_{l}^{k+1}\right)}+\sqrt{\varepsilon\left(x_{r}^{k}\right)}\right) \mathcal{F}_{l}^{k+1} \hat{\boldsymbol{u}}_{h}^{k+1} \\
& -\frac{1}{2}\left(\sqrt{\varepsilon\left(x_{r}^{k-1}\right)}+\sqrt{\varepsilon\left(x_{l}^{k}\right)}\right) \mathcal{F}_{l}^{k} \hat{\boldsymbol{u}}_{h}^{k},
\end{aligned}
$$

where $\hat{\boldsymbol{q}}_{h}^{k}$ is the vector of degrees of freedom associated to the auxiliary variable $q$ and $\mathcal{S}^{\sqrt{\varepsilon}}$ and $\tilde{\mathcal{S}}^{\sqrt{\varepsilon}}$ are modified stiffness matrices which take into account the artificial viscosity function $\varepsilon(x)$ [7]. It is worth noting that as $\hat{\boldsymbol{f}}_{h}^{k}$, the vector $\hat{\boldsymbol{q}}_{h}^{k}$ is also locally computed.

\section{SHOCK CAPTURING SCHEMES}

As a typical nonlinear PDE based on a conservation law, the LWR equation naturally develops shocks along time integration. When high order discretization techniques such as DGFEM are used to approximate solutions for this equation, stabilizing schemes are necessary to control undesired oscillations in the vicinity of discontinuities due to the Gibbs phenomenon.

We chose two standard approaches to stabilize the numerical solutions computed by the high order DGFEM solver: limiters and sub-cell shock capturing based on artificial viscosity adding. In the first case we implemented a generalized slope limiter $\left(\Pi^{N}\right)$ as described in [8] and 
a hierarchical slope limiter proposed in [9]. In the second approach we implemented the subcell shock capturing scheme as originally proposed in [11] which uses elementwise constant artificial viscosity. We also implemented a modified version of this strategy which considers the artificial viscosity as a $C^{0}$ function [12]. The details of the mathematical formulation of the sub-cell shock capturing scheme applied to the LWR traffic flow equation can be found in [7].

\section{NUMERICAL EXPERIMENTS}

The LWR equation was approximated considering three different initial conditions: (1) Red light at the exit of the road; (2) Green light at half way of the road length and (3) Exponential density configuration at the entrance of the road. All examples were set with appropriate Dirichlet or Neumann boundary conditions. For each of these initial conditions the numerical solutions were marched in time for 2.0 minutes.

The five step $4^{\text {th }}$ order strong stability preserving Runge-Kutta explicit integrator suggested in [8] was used to evolve the semi-discrete formulation (equations 9 and 14) in time.

We organized our experiments in four subsections: the first subsection presents the overall aspect of the numerical solution of the LWR equation for the three initial conditions considered. For each initial condition, we depict the behavior of the four shock capturing schemes we have chosen. In the second subsection, we compare the performance of both limiters, namely, hierarchical slope limiter (HL) and generalized slope limiter (GSL) in isolation when increasing the number of grid elements $(K)$ and polynomial order $(N)$. Likewise, in the third subsection, we compare the performance of both stabilizing schemes based on artificial viscosity, namely, sub-cell shock capturing with elementwise constant artificial viscosity (Constant-SCSC) and sub-cell shock capturing with local $C^{0}$ artificial viscosity ( $C^{0}$-SCSC) in isolation in terms of the number of grid elements and polynomial order. Finally, we compare the two schemes showing the better performance (limiter vs. artificial viscosity) in terms of the number of degrees of freedom (NDOF) to achieve an acceptable resolution of the jump discontinuity.

\subsection{Traffic initial condition examples}

\subsubsection{Red light}

For the red light example a traffic light indicating stop is positioned at the end of a road of $4 \mathrm{Km}$ long. Cars accumulate quickly near the road exit, where we have the maximum allowed density of cars between 3 and $4 \mathrm{Km}$. There is an incoming traffic of $50 \%$ the maximum allowed density $\left(u=0.5 u_{\max }\right)$. This is a standard benchmark problem for the LWR equation because it has an exact solution given by a moving shock wave traveling backward from the exit to the entrance of the road. The remaining parameters of the problem were defined as follows: car speed limit $V_{\max }=1.0 \mathrm{Km} / \mathrm{min}$, and maximum car density per unit length $u_{\max }=10.0 \mathrm{cars} / \mathrm{Km}$. Non homogeneous Dirichlet boundary conditions were set at both limits of the road with the following values: $u(x=0)=5.0$ cars $/ K m$, at left, and, $u(x=$ 4) $=10.0$ cars $/ \mathrm{Km}$, at right.

The red light problem solutions were stabilized with the four shock capturing schemes described in section 4 . For limiters, we set the number of grid elements and the polynomial order of approximation as $K=500$ and $N=2$. In the case of artificial viscosity based schemes, we set $K=20$ and $N=10$. Figure 2 depicts the 3D graphics of the numerical solution of this problem for the four shock capturing schemes.

We can clearly notice that the Constant-SCSC scheme produced many spikes over the numerical solution in different time instants. The other schemes showed very similar solution 

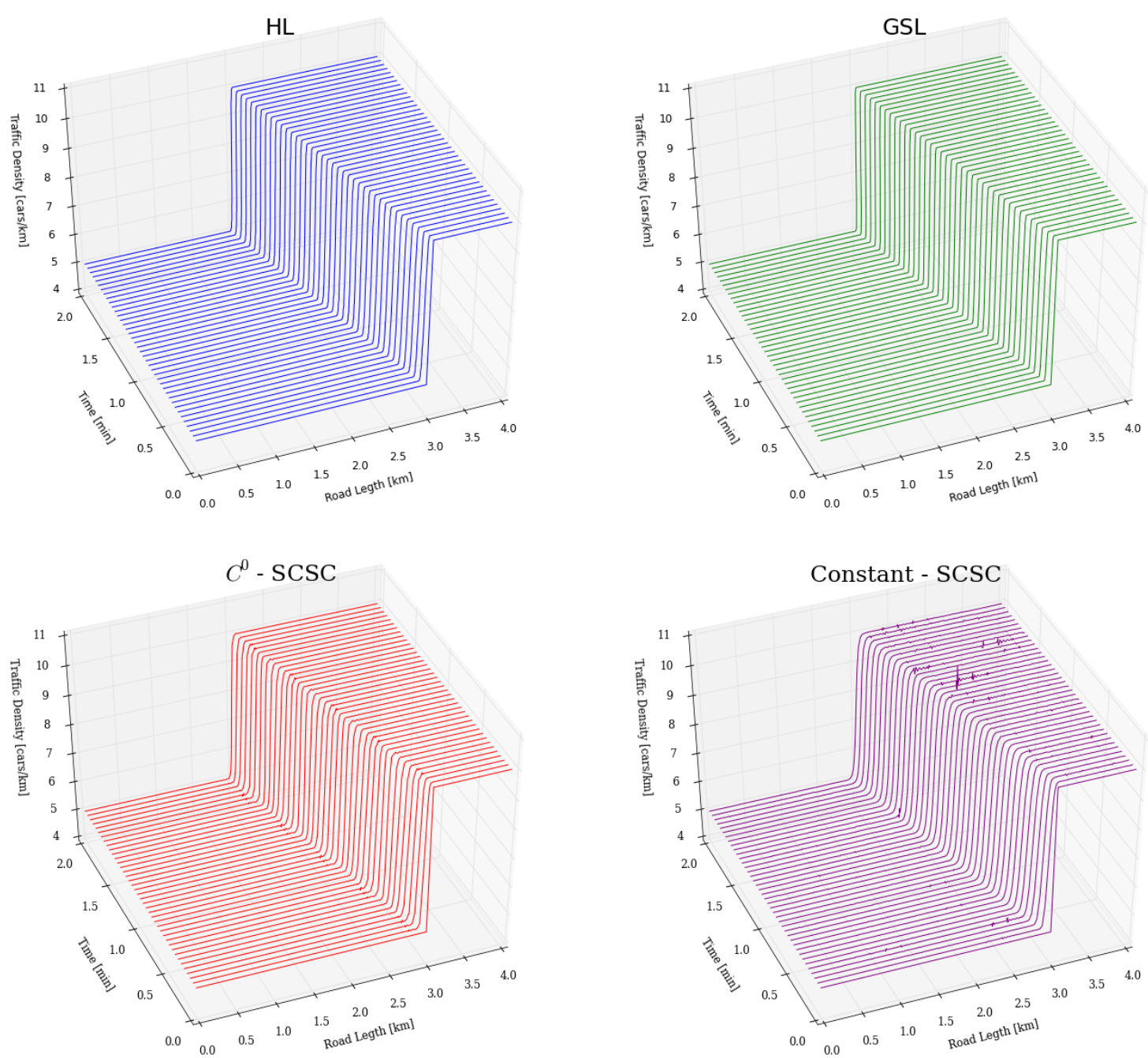

Figure 2: Red Light problem solutions using the four shock capturing schemes.

profiles.

\subsubsection{Green light}

For the green light example we suppose there exists a traffic light at the half way of a road of $4 \mathrm{Km}$ long where cars are bumper-to-bumper. The traffic density decreases linearly to zero towards the entrance of the road and ahead of the traffic light the road is clear. When the traffic light turns green cars start to move and the car density profile changes its original triangular shape. The car density profile starts with a jump discontinuity at the wave front and develops a shock at the rear of the propagating wave. For this example, the following setup parameters were used: car speed limit $V_{\max }=1.0 \mathrm{Km} / \mathrm{min}$, and maximum car density per unit length $u_{\max }=10.0 \mathrm{cars} / \mathrm{Km}$. Homogeneous Dirichlet boundary conditions were set at both limits of the road.

As in the previous example, numerical solutions were stabilized with the four shock capturing schemes and the following configurations were set for limiters: $K=500$ and $N=2$, and for artificial viscosity based schemes: $K=20$ and $N=10$. Figure 3 depicts the 3D graphics of the numerical solution of the green light problem for the four shock capturing schemes. 

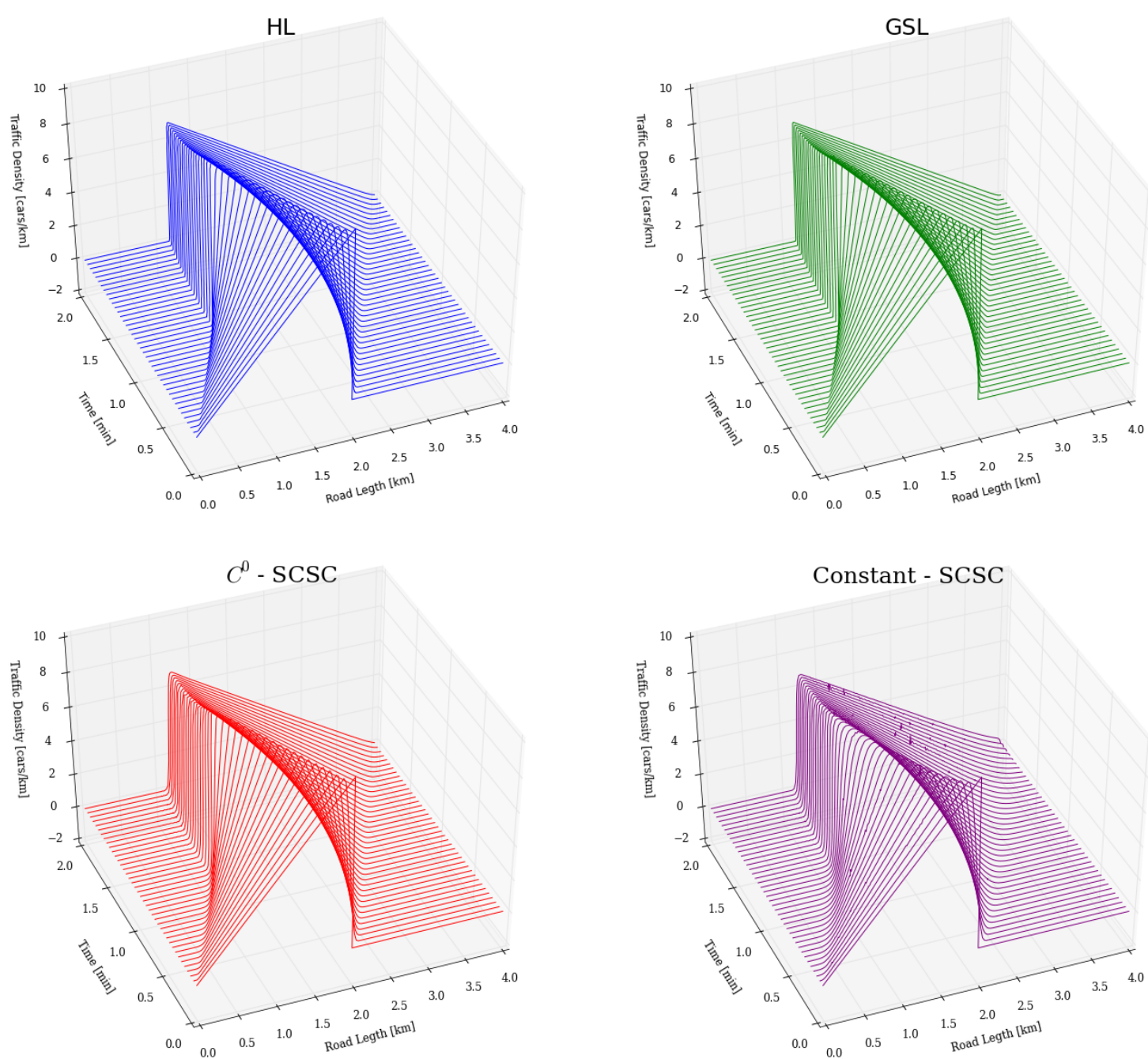

Figure 3: Green light problem solutions using the four shock capturing schemes.

The same observations made for the numerical solutions of the red light example are valid for the green light problem.

\subsubsection{Exponential}

The exponential problem is a rather theoretical case study for the LWR equation which shows a shock formation from a smooth initial profile. In this case, there is no traffic light along a road of $2 \pi K m$ long. The remaining setup parameters were chosen as follows: speed limit $V_{\max }=1.0 \mathrm{Km} / \mathrm{min}$, and maximum traffic density $u_{\max }=1.0 \mathrm{car} / \mathrm{Km}$. At left, it was set a non homogeneous time dependent Dirichlet boundary condition given as $u(t)=1.0+\sin (t)$ and, at right, a homogeneous Neumann boundary condition was prescribed.

Again, we can see in the figure 4 the 3D graphics representing the numerical solution of the exponential problem for the four shock capturing schemes. In this case, again, the following setups were fixed for limiters: $K=500$ and $N=2$, and for artificial viscosity based schemes: $K=20$ and $N=10$.

For this example, all the numerical solutions revealed very similar shapes and no spikes could be noticed over the solution stabilized by the Constant-SCSC scheme, at least visually. 

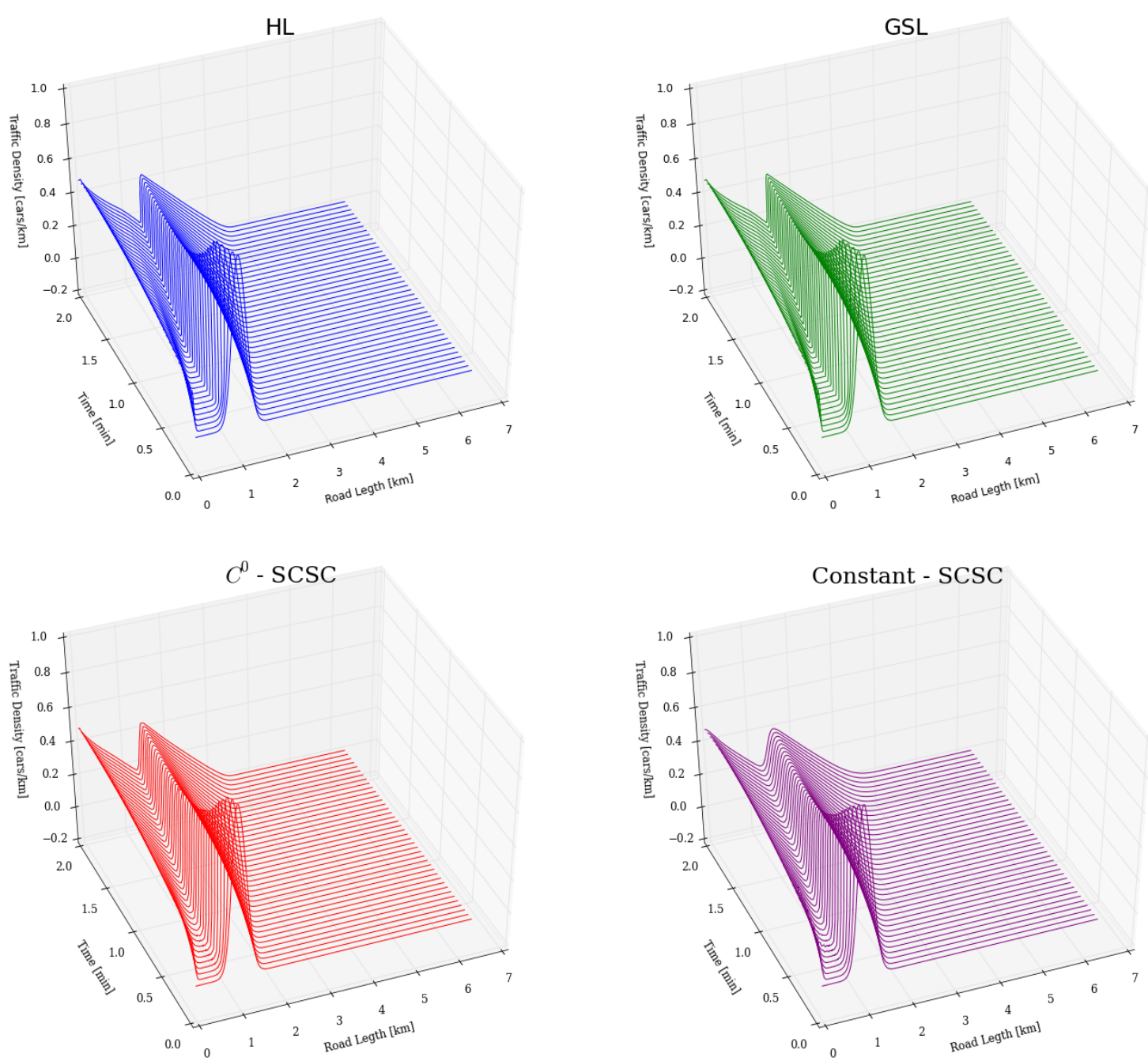

Figure 4: Exponential problem solutions using the four shock capturing schemes.

\subsection{Limiters}

This subsection aims at checking the ability of each slope limiter, HL and GSL, to improve shock resolution when increasing the number of grid elements, $K$, and the polynomial order, $N$. For each traffic problem studied, these quantities were fixed as $K=100,170,240,300$, and $N=2,6,10$. Snapshots of the shock formation were taken at $t=1.2,1.0,0.7 \mathrm{~min}$ for the red light, green light and exponential examples, respectively. 


\subsubsection{Hierarchical slope limiter}

Figures 5, 6 and 7 depicts the performance of the hierarchical slope limiter as a function of the total number of grid elements and the polynomial order of approximation.
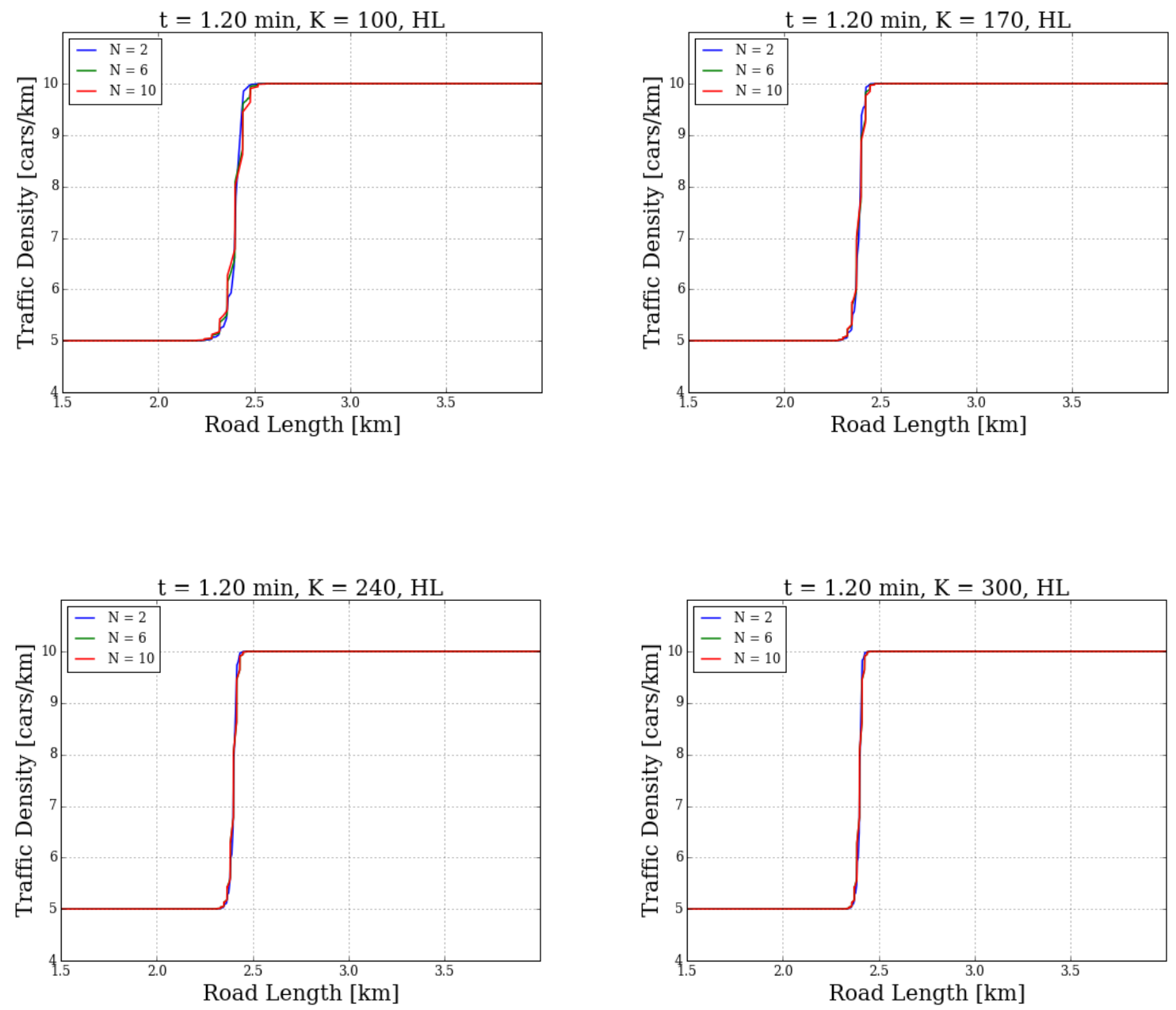

Figure 5: Red Light initial condition.
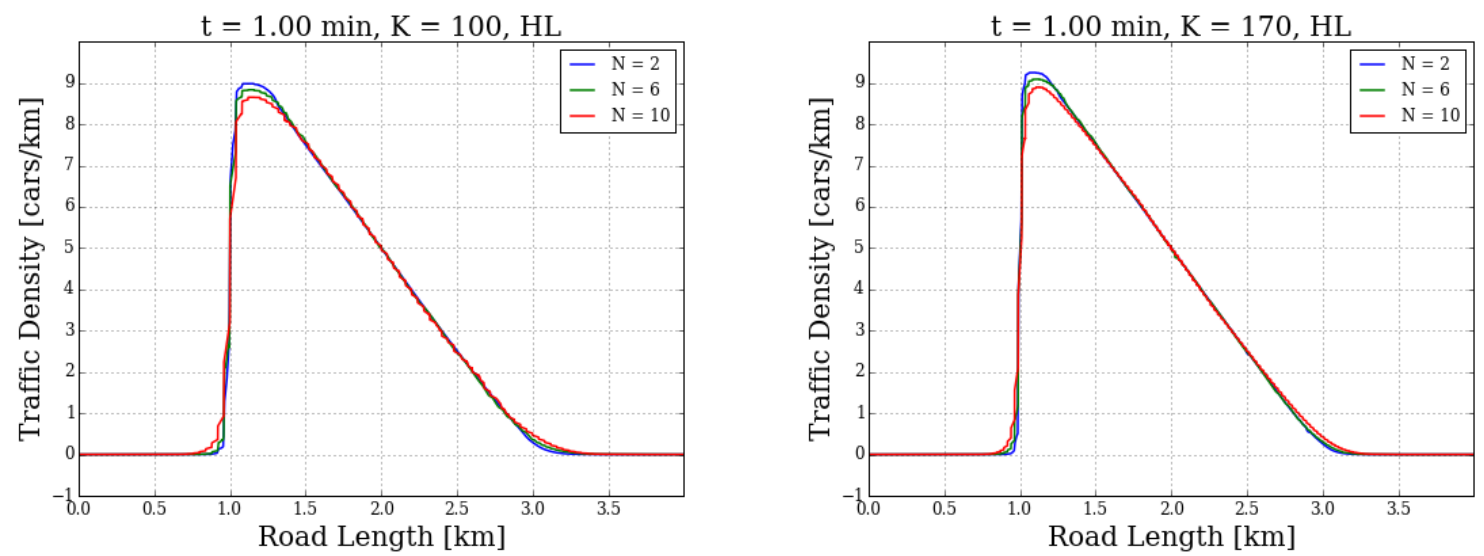

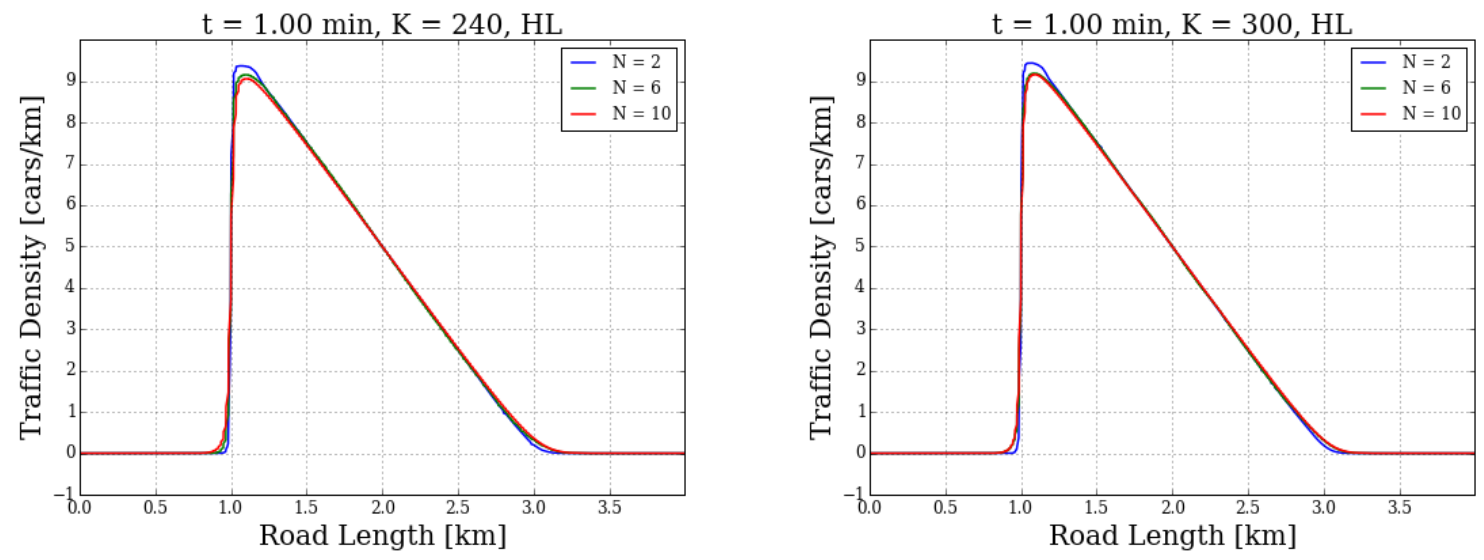

Figure 6: Green Light initial condition.
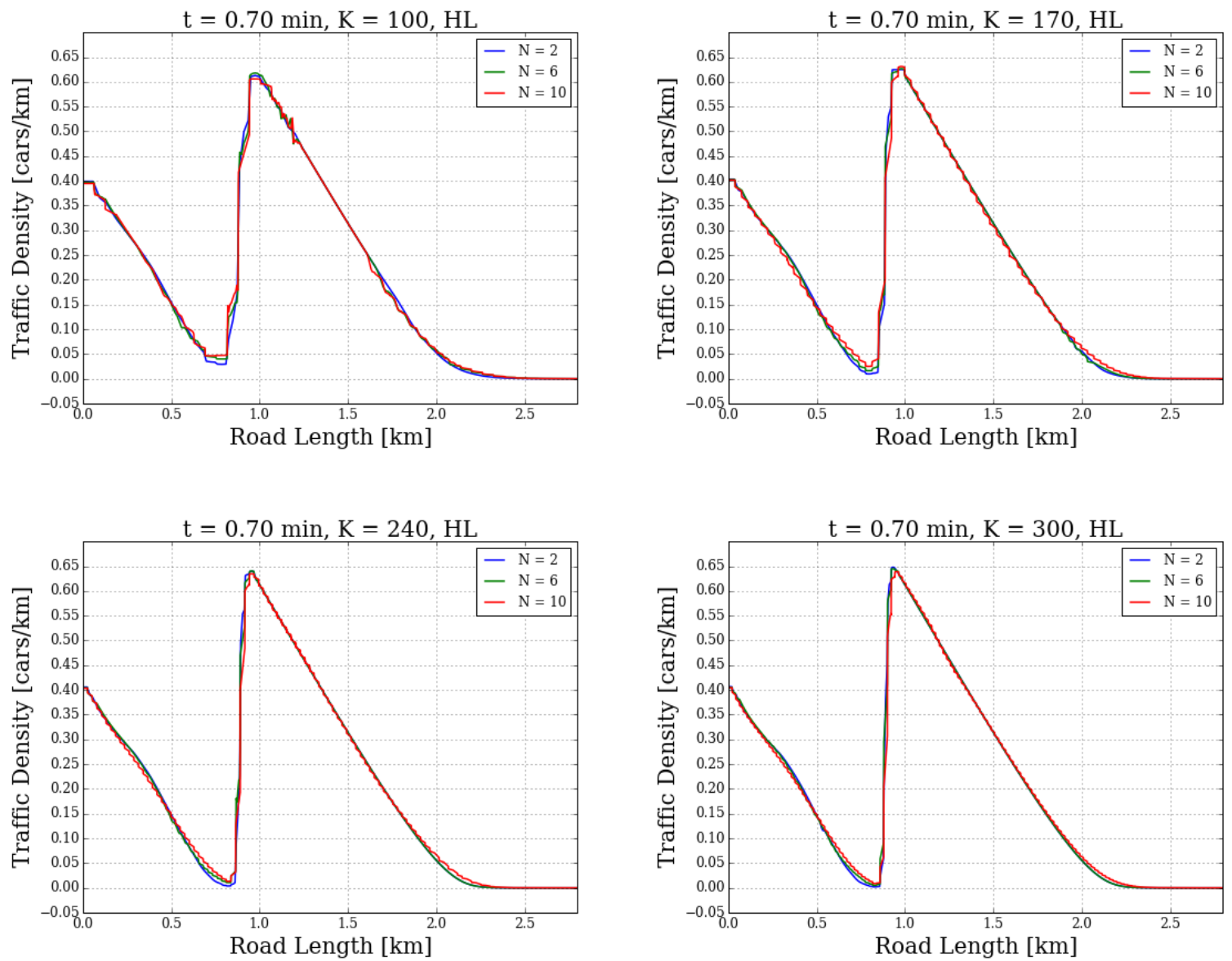

Figure 7: Exponential initial condition.

We can observe from these graphics that the increase of grid elements rather than the polynomial order is the best way to improve the shock resolution. Moreover, we can note that the greater the polynomial order the worse the overall quality of the solutions for a fixed number of elements. 


\subsubsection{Generalized slope limiter}

Figures 8, 9 and 10 depicts the performance of the generalized slope limiter $\left(\Pi^{N}\right)$ as a function of the total number of grid elements and the polynomial order of approximation.
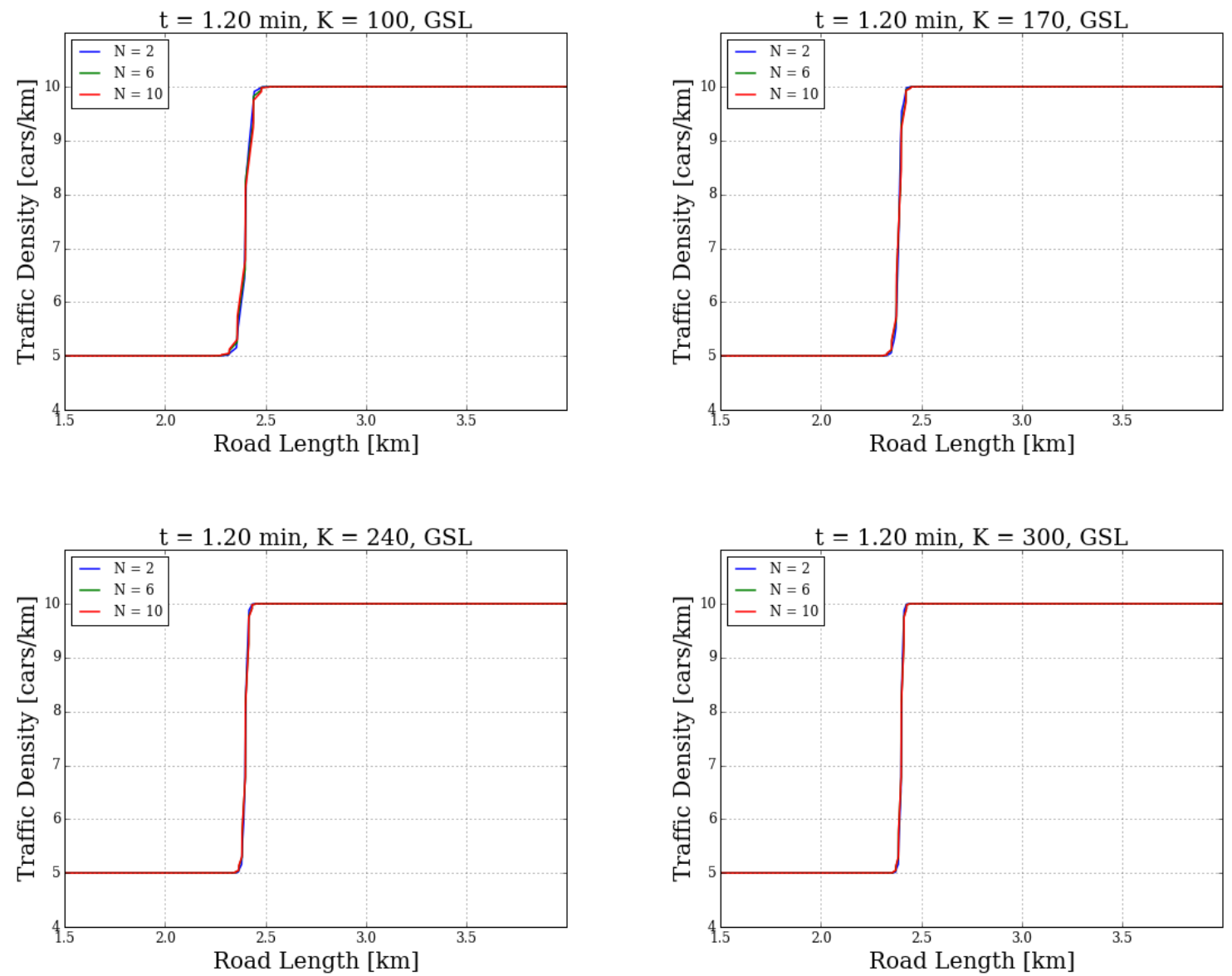

Figure 8: Red Light initial condition
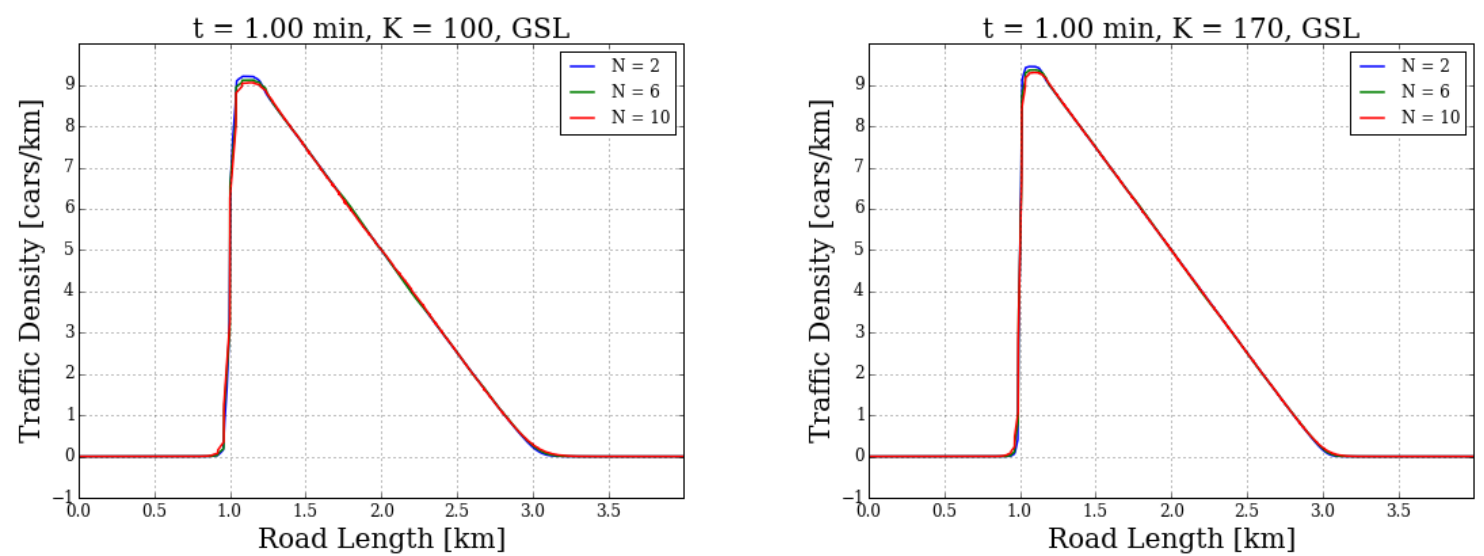

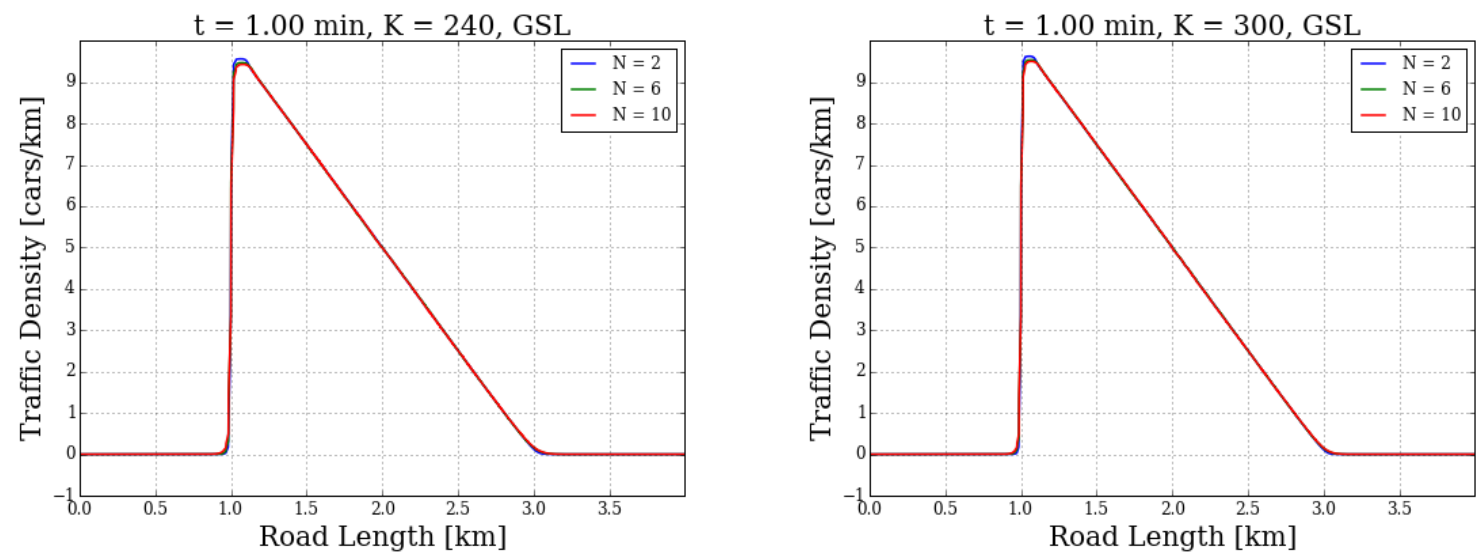

Figure 9: Green Light initial condition
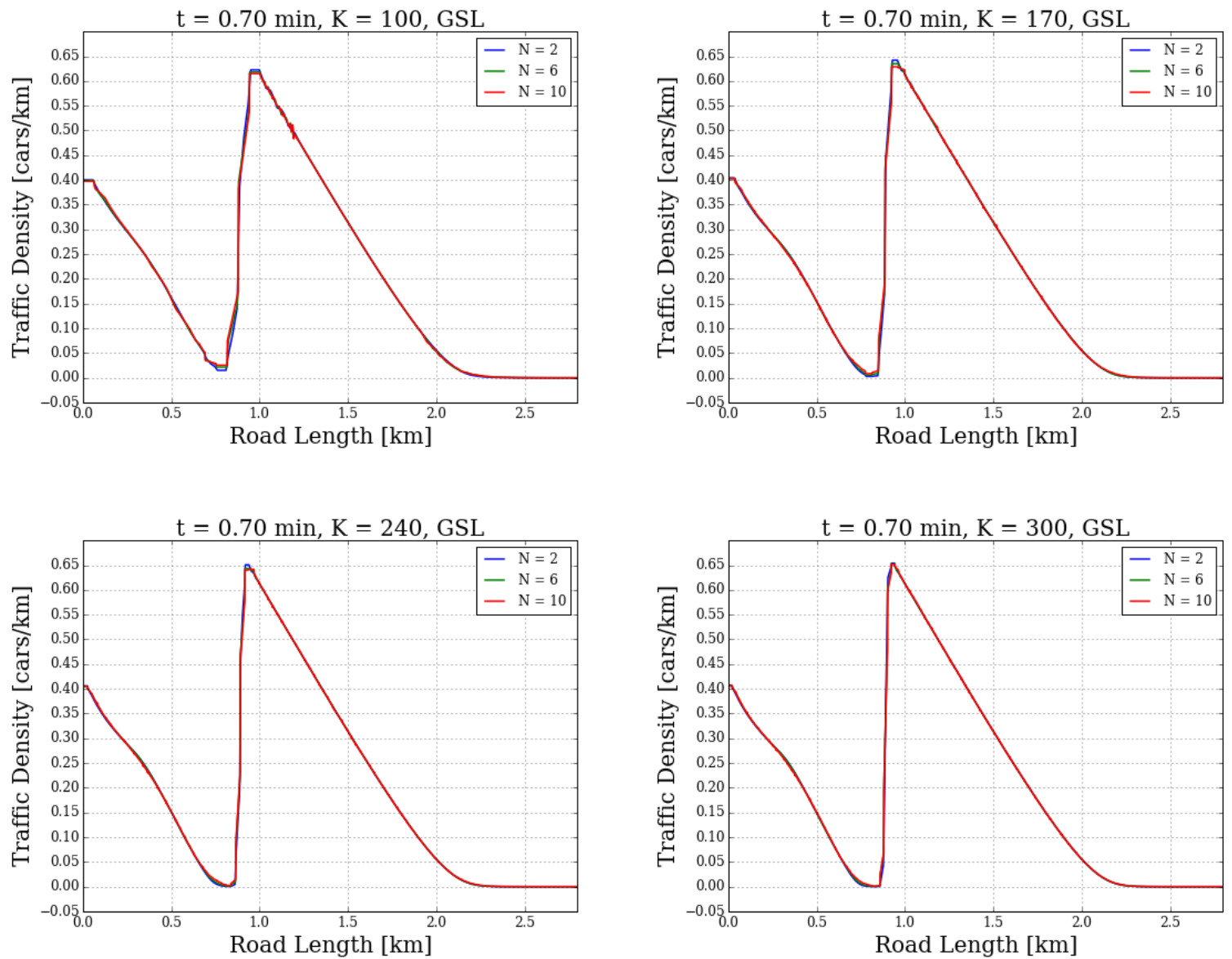

Figure 10: Exponential initial condition.

We can notice that the GSL showed better resolution capabilities when using a large number of elements as in the case of the HL despite being less sensitive to polynomial order increment. Our goal with this first set of experiments was to prove that limiters are not greatly affected by the increment of polynomial order to display sharp shock resolution requiring quite refined grids to achieve reasonable sharpness. 


\subsection{Sub-cell shock capturing schemes}

As in the previous study using limiters, this subsection intends to verify the ability of the artificial viscosity based schemes, Constant-SCSC and $C^{0}$-SCSC, to improve shock resolution as a function of the number of grid elements, $K$, and the polynomial order, $N$. We fixed $K=40$ for the Constant-SCSC scheme and $K=20$ for the $C^{0}$-SCSC scheme as the former scheme required more degrees of freedom to show minimally acceptable results. For both schemes, the polynomial order were set as $N=2,6,10$. Again, snapshots of the shock formation were taken at $t=1.2,1.0,0.7 \mathrm{~min}$ for the red light, green light and exponential examples, respectively. It is worth mention that sub-cell shock capturing strategies required much smaller time steps than limiters to ensure stable solutions. Overall, limiters used four times larger time steps than sub-cell shock capturing schemes.

\subsubsection{Constant-SCSC}

Figures 11, 12 and 13 depicts the overall performance of the constant-SCSC scheme as a function of the total number of grid elements and the polynomial order of approximation.
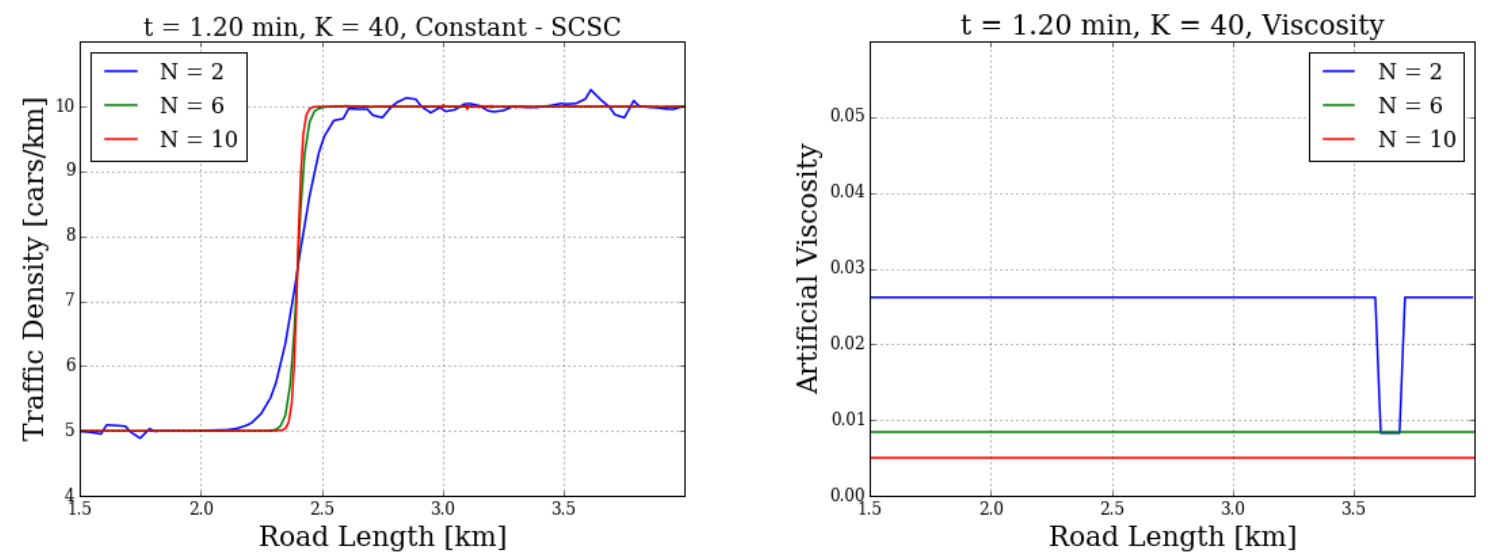

Figure 11: Red Light initial condition
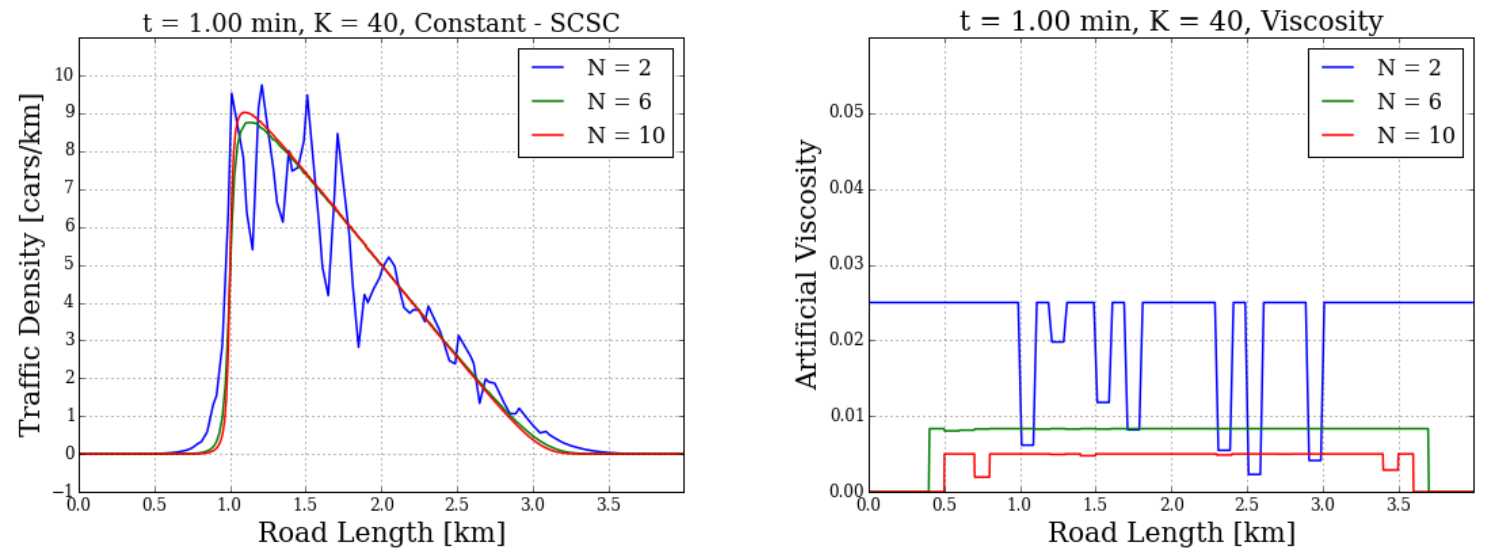

Figure 12: Green Light initial condition 

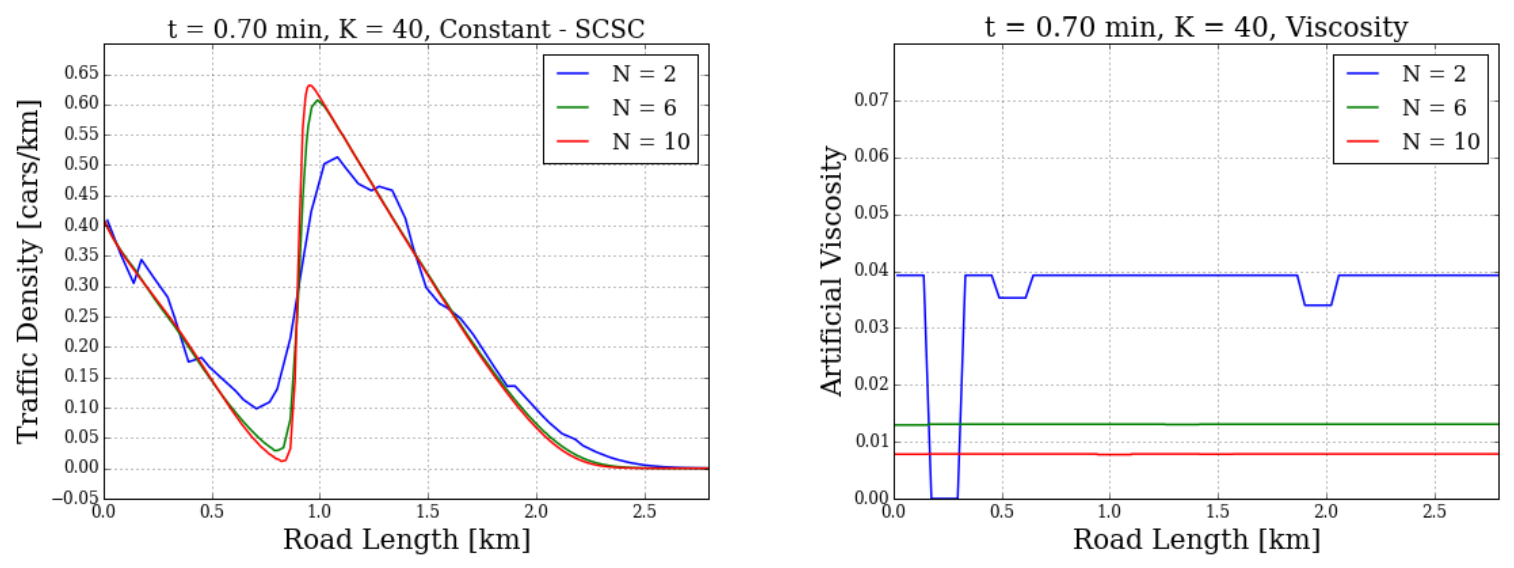

Figure 13: Exponential initial condition

From the above mentioned figures, it is evident that the sub-cell shock capturing scheme based on a globally discontinuous viscosity function $\varepsilon(x)$ produced very poor quality results for low polynomial orders. Even with high order polynomial approximations such scheme developed small traveling spikes over the solution profiles destroying the locality of the method and resulting in excessive smearing of the jump discontinuities.

\subsection{2 $C^{0}-$ SCSC}

Figures 14, 15 and 16 depicts the overall performance of the $C^{0}$-SCSC scheme as a function of the total number of grid elements and the polynomial order of approximation.
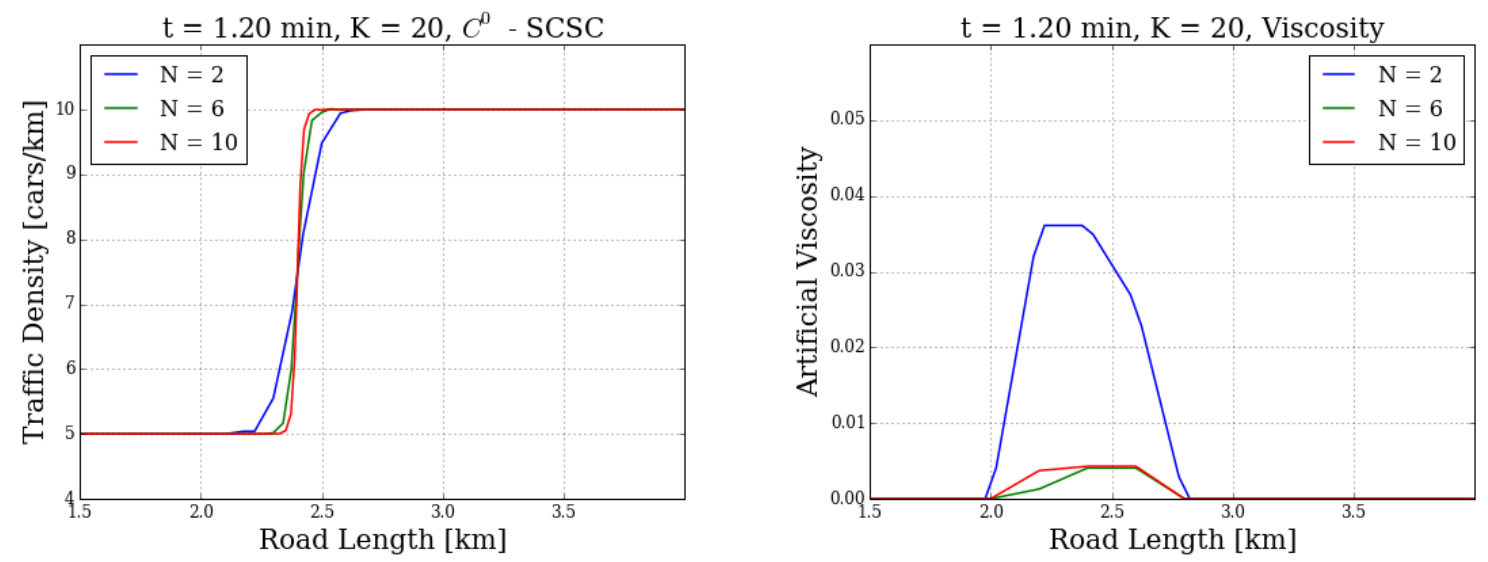

Figure 14: Red Light initial condition

Contrary to what has been revealed by the Constant-SCSC approach, the $C^{0}$-SCSC scheme demonstrated very reliable even for very coarse grids. Sharpness in shock resolution was clearly improved with polynomial order increasing and the locality of the added artificial viscosity was definitely proved. This second set of experiments aimed at showing that artificial viscosity mechanisms perform much better with very high order approximations and larger elements, and high resolution of the shock representation is achieved with the increment of the polynomial order. 

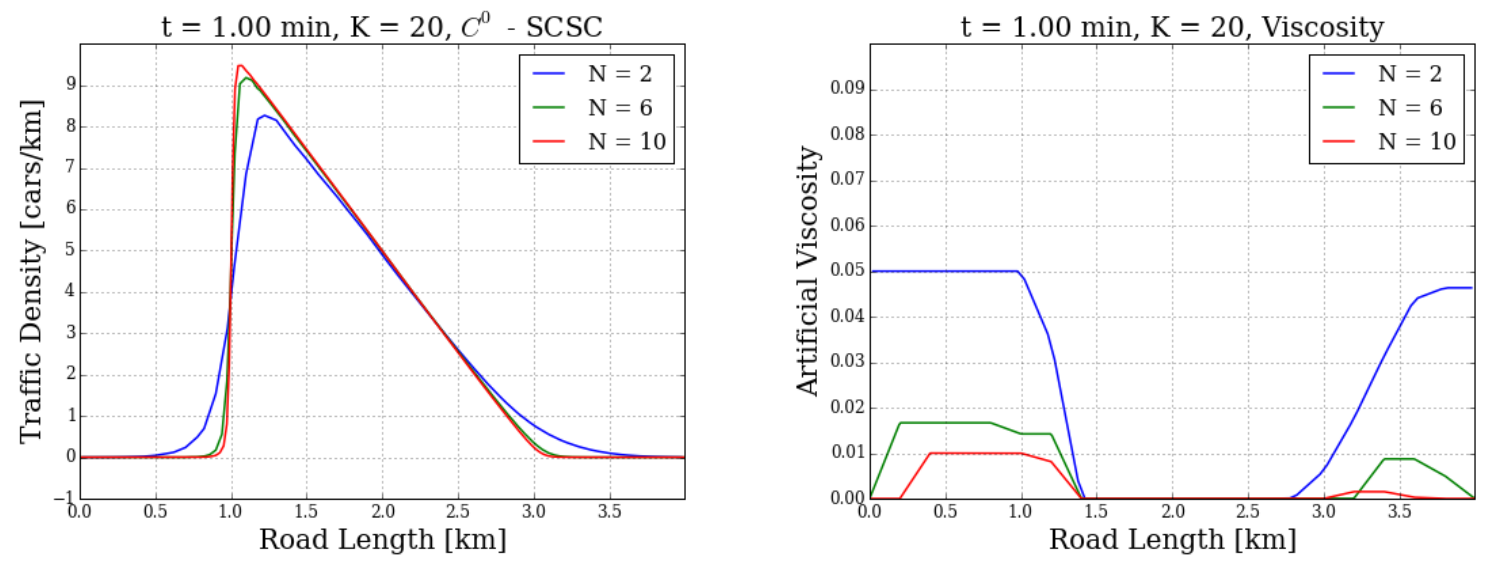

Figure 15: Green Light initial condition
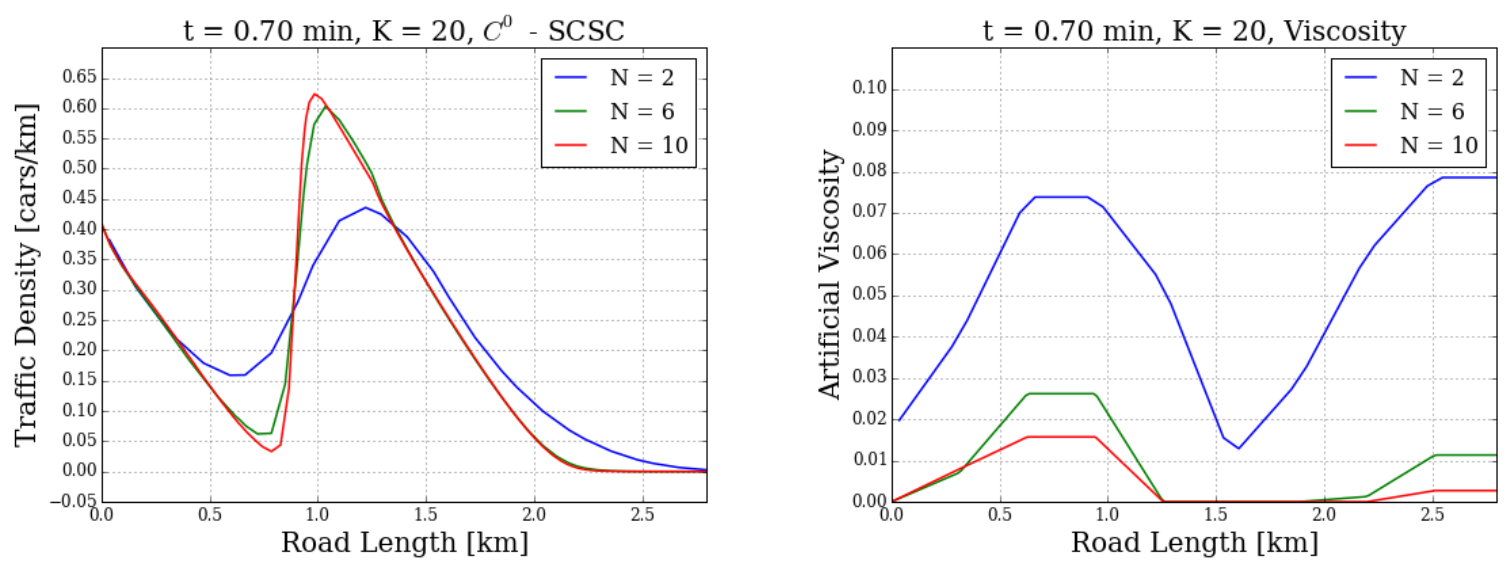

Figure 16: Exponential initial condition

\subsection{Limiter vs. Sub-cell shock capturing}

This subsection was designed to evaluate which shock capturing scheme shows the best cost-benefit to sharply resolve the shock formation using an unbiased criterion to measure computational performance. To achieve such goal, we compare GSL to $C^{0}$-SCSC in terms of the total degrees of freedom required by each scheme to reach a reasonable resolution of jump discontinuities.

Figures 17, 18 and 19 compare the overall performance of the GSL and the $C^{0}$-SCSC schemes as a function of the total number of degrees of freedom. Snapshots of the shock formation were taken at $t=1.2,1.0,0.7 \mathrm{~min}$ for the red light, green light and exponential examples, respectively. The number of elements and the polynomial orders are indicated in the caption of each figure for both schemes. For GSL we kept $N=1$ and increased the number of elements $K$ as we have previously demonstrated the weak impact of polynomial order in the shock resolution. In the case of $C^{0}$-SCSC, we increased $N$ as much as we reduced $K$ once the most remarkable feature of this scheme is to achieve sharp shock resolution with very coarse grids and high order polynomial approximations.

Results from both techniques showed very similar for the red light example. With just a few degrees of freedom to approximate the solution, $C^{0}$-SCSC was very dissipative but GSL 


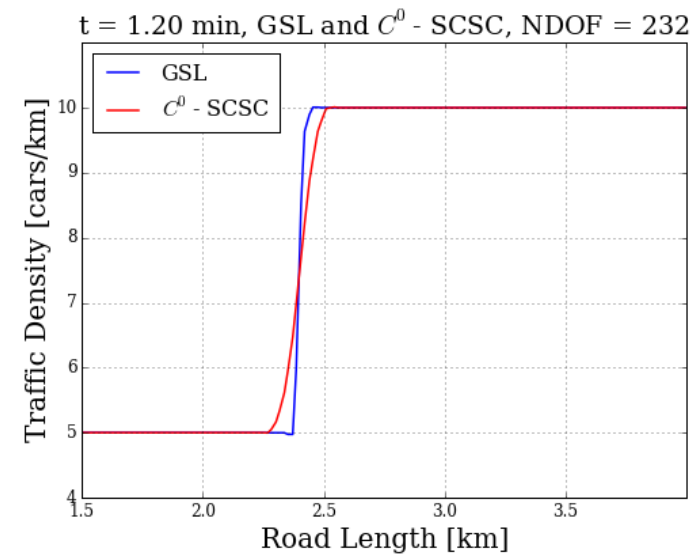

(a) GSL: $\mathrm{K}=116, \mathrm{~N}=1 . C^{0}-\mathrm{SCSC}: \mathrm{K}=116, \mathrm{~N}=1$

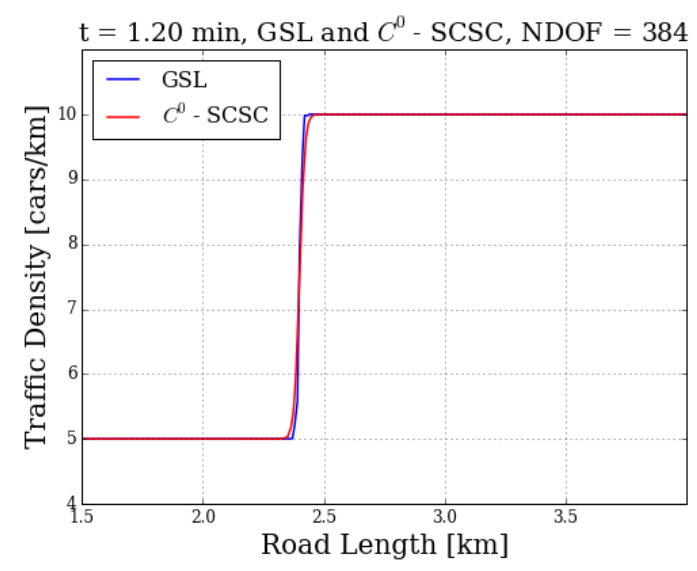

(c) GSL: $\mathrm{K}=192, \mathrm{~N}=1 . C^{0}-\mathrm{SCSC}: \mathrm{K}=64, \mathrm{~N}=5$

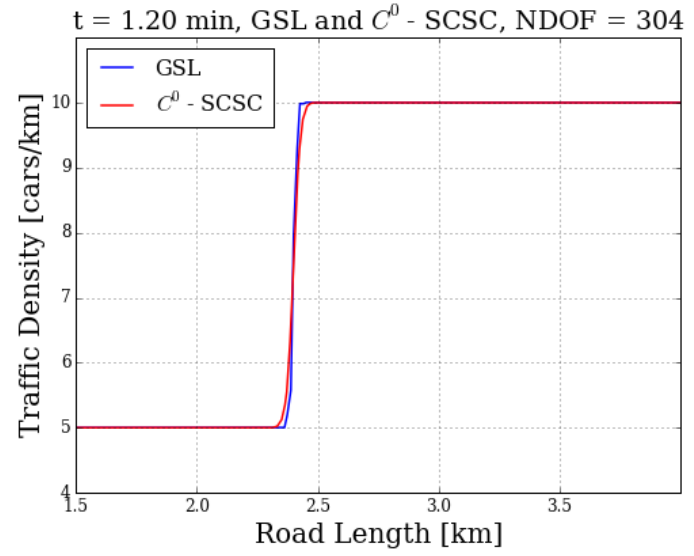

(b) GSL: $\mathrm{K}=152, \mathrm{~N}=1 . C^{0}-\mathrm{SCSC}: \mathrm{K}=76, \mathrm{~N}=3$

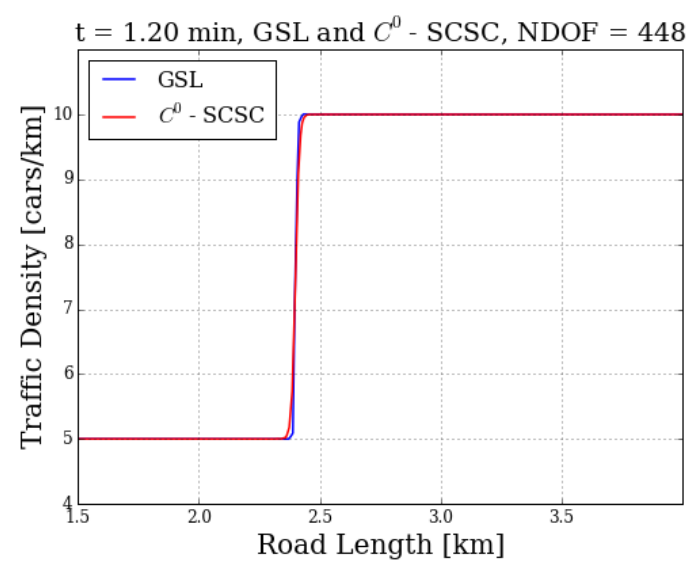

(d) GSL: $\mathrm{K}=224, \mathrm{~N}=1 . C^{0}-\mathrm{SCSC}: \mathrm{K}=56, \mathrm{~N}=7$

Figure 17: Red light initial condition

showed a small oscillation at the base of the discontinuity. For the maximum number of degrees of freedom, GLS seemed to slightly trim the sharp corners of the shock.

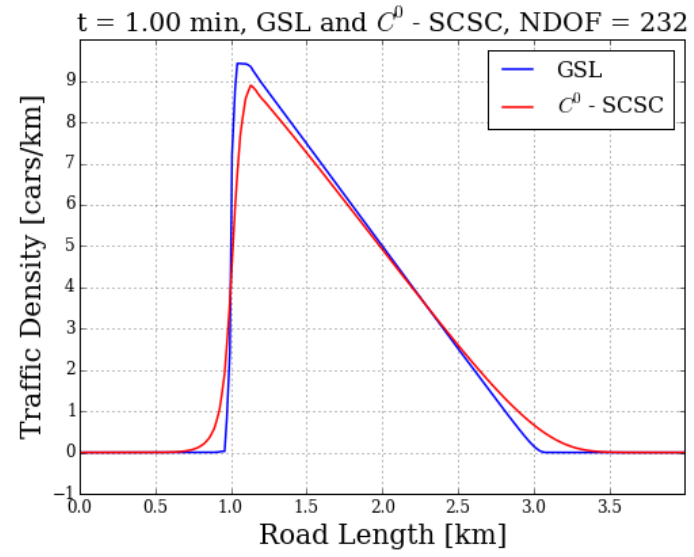

(a) GSL: $\mathrm{K}=116, \mathrm{~N}=1 . C^{0}-\mathrm{SCSC}: \mathrm{K}=116, \mathrm{~N}=1$

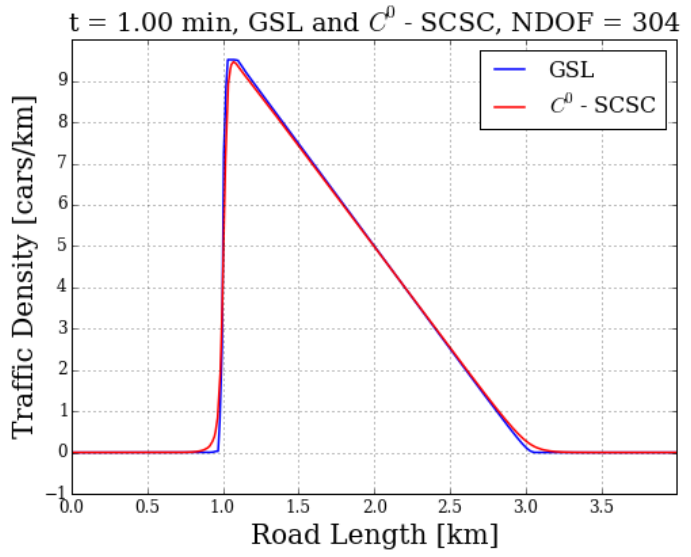

(b) GSL: $\mathrm{K}=152, \mathrm{~N}=1 . C^{0}-\mathrm{SCSC}: \mathrm{K}=76, \mathrm{~N}=3$ 


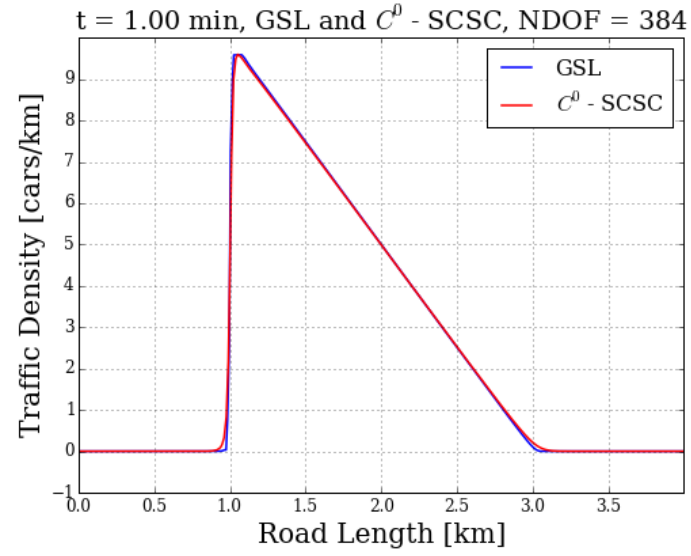

(c) GSL: $\mathrm{K}=192, \mathrm{~N}=1 . C^{0}-\mathrm{SCSC}: \mathrm{K}=64, \mathrm{~N}=5$

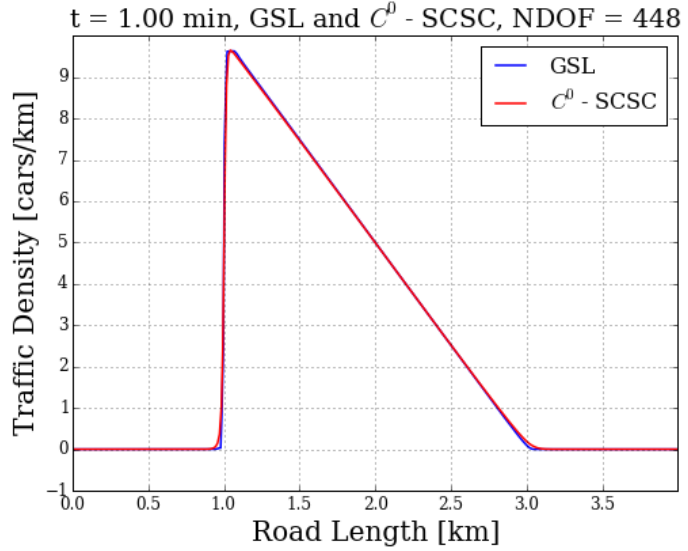

(d) GSL: $\mathrm{K}=224, \mathrm{~N}=1 . C^{0}-\mathrm{SCSC}: \mathrm{K}=56, \mathrm{~N}=7$

Figure 18: Green light initial condition

For the green light example, GSL trimmed the top corner of the shock in every case. As expected, $C^{0}$-SCSC solutions were slightly more dissipative but still able to sharply characterize the shock resolution.

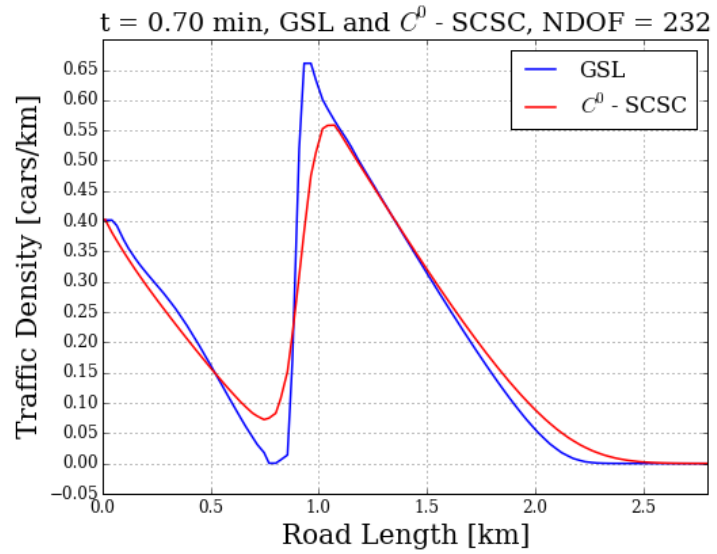

(a) GSL: $\mathrm{K}=116, \mathrm{~N}=1 . C^{0}-\mathrm{SCSC}: \mathrm{K}=116, \mathrm{~N}=1$

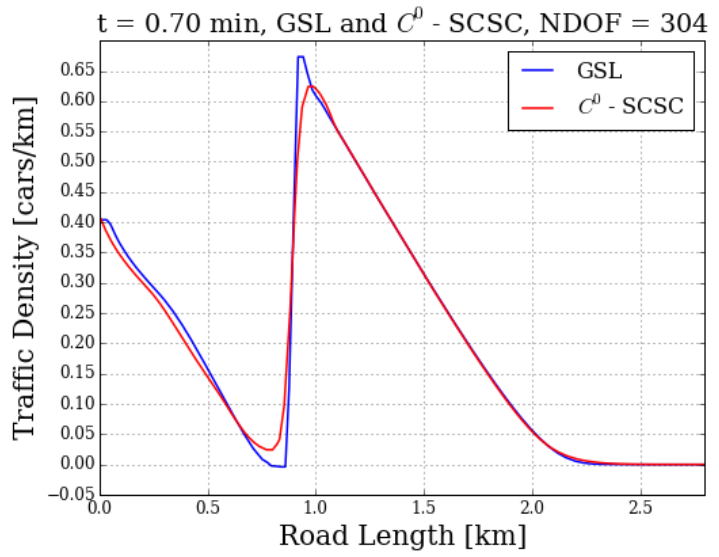

(b) GSL: $\mathrm{K}=152, \mathrm{~N}=1 . C^{0}-\mathrm{SCSC}: \mathrm{K}=76, \mathrm{~N}=3$

For the exponential example, $C^{0}$-SCSC scheme proved superior as GSL was not able to control instabilities at the base of the jump discontinuity and also trimmed the top corner of the shock in every situation. As already observed, the $C^{0}$-SCSC scheme worked quite well for very high order approximations. 


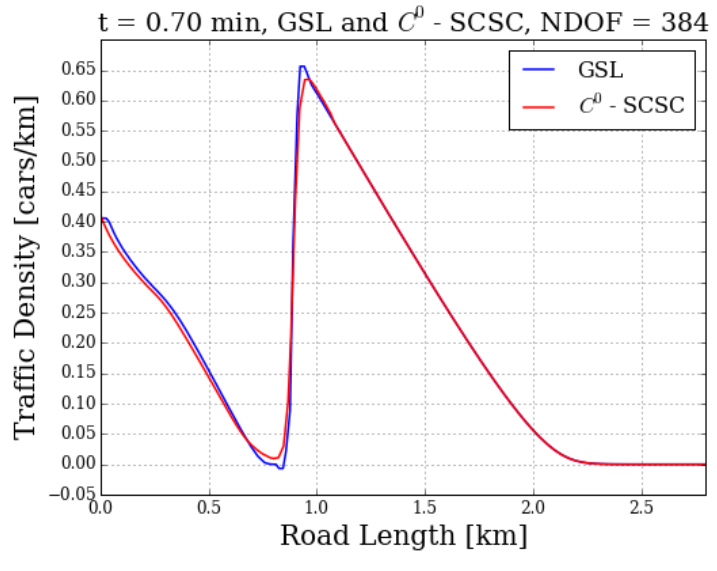

(c) GSL: $\mathrm{K}=192, \mathrm{~N}=1 . C^{0}-\mathrm{SCSC}: \mathrm{K}=64, \mathrm{~N}=5$

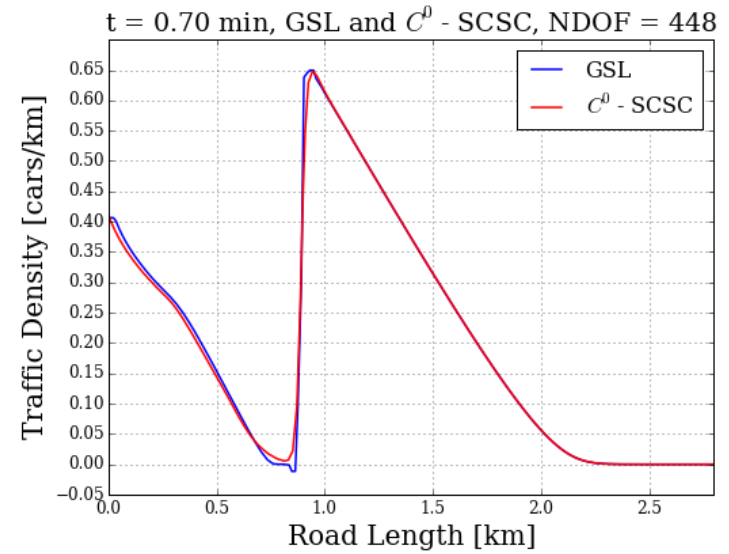

(d) GSL: $\mathrm{K}=224, \mathrm{~N}=1 . C^{0}-\mathrm{SCSC}: \mathrm{K}=56, \mathrm{~N}=7$

Figure 19: Exponential initial condition.

\section{CONCLUSIONS}

This paper discussed the implementation and verification of four different strategies to stabilize the numerical solution of the LWR equation discretized with a modal version of the high order discontinuous Galerkin method. We selected two efficient limiters (HL and GSL) [8, 9] as well as two artificial viscosity based schemes (Constant-SCSC and $C^{0}$-SCSC) to stabilize our numerical solutions. We proposed three examples of initial conditions to test our implementations: (a) red light at road exit; (b) green light at half way of road length and (c) exponential car density at road entrance.

We were able to prove that limiters are not greatly affected by the increment of polynomial order to display sharp shock resolution requiring quite refined grids to achieve reasonable results. The HL revealed more sensitive to polynomial order refinement as it produced poorer results when compared to the GSL for high order approximations.

Both sub-cell shock capturing schemes demonstrated good robustness to sharply represent shock structures with very coarse grids. High resolution was achieved for increasing polynomial order. However, it is worth remarking that sub-cell shock capturing with elementwise constant artificial viscosity produced traveling spikes over the solution profiles during time integration causing the shock sensor activation most of the spatial domain and therefore destroying the acclaimed locality of the method. It also resulted in excessive smearing of the shock structure.

Sub-cell shock capturing with $C^{0}$ artificial viscosity [12] proved very reliable to stabilize solutions in the presence of jump discontinuities although it required smaller time steps for explicit time integration. Such stabilizing scheme displayed the most noticeable results exhibiting sharp shock resolution features in very coarse meshes with very little dissipation. Overall, such scheme revealed the best cost-benefit in terms of computational efficiency based on the number of degrees of freedom for a certain level of shock resolution.

\section{ACKNOWLEDGEMENTS}

The authors would like to thank the IBM Research Brazil for the technical and financial support which enabled the development of this research initiative. 


\section{REFERENCES}

[1] M.J. Lighthill, G.B. Whitham, On kinematic waves II. A theory of traffic flow on long crowded roads, Proceedings of the Royal Society of London. Series A, Mathematical and Physical Sciences, 229(1178), 317-345, 1955.

[2] P.I. Richards, Shock waves on the highway, Operations Research, 4(1), 42-51, 1956.

[3] W. Ceulemans, M.A. Wahab, K. De Proft, G. Wets, Modelling Traffic Flow with Constant Speed using the Galerkin Finite Element Method. Proceedings of the World Congress on Engineering, Vols. I and II, 993-999, 2009.

[4] C. Suncica, B. Piccoli, J.M. Qiu, T. Ren, Runge-Kutta Discontinuous Galerkin Method for Traffic Flow Model on Networks. ArXiv e-prints, 1403.3750, math.NA, 2014.

[5] B.D. Greenshields, A study of highway capacity. Highway Research Board Proceedings, 14, 448-477, 1935.

[6] G.E. Karniadakis, S.J. Sherwin, Spectral/hp Element Methods for Computational Fluid Dynamics, $2^{\text {nd }}$ Edition. Oxford University Press, 2005.

[7] A.C. Nogueira Jr., J. Bernardo, S. Moore. Solution of the LWR Nonlinear Traffic Flow Model using a Discontinuous Galerkin FEM Approach. Proceedings of the CILAMCE XXXVI - 36th Ibero-Latin American Congress on Computational Methods in Engineering, Rio de Janeiro, Brazil, Nov. 22-25, 2015.

[8] J.S. Hesthaven, T. Warburton, Nodal Discontinuous Galerkin Methods: Algorithms, Analysis, and Applications. Springer, 2008.

[9] L. Krivodonova, Limiters for high-order discontinuous Galerkin methods. Journal of Computational Physics, 226, 879-896, 2007.

[10] B. Cockburn, C.-W. Shu, The local discontinuous Galerkin method for time-dependent convection-diffusion systems. SIAM Journal on Numerical Analysis, 35, 2440-2463, 1998.

[11] P-O. Persson, J. Peraire, Sub-Cell Shock Capturing for Discontinuous Galerkin Methods. Proceedings of the $44^{\text {th }}$ AIAA Aerospace Sciences Meeting and Exhibit, 112, 2006.

[12] A. Klöckner, T. Warburton, J.S. Hesthaven. Viscous Shock Capturing in a Time-Explicit Discontinuous Galerkin Method. Mathematical Modelling of Natural Phenomena, 6(3), 57-83, 2011. 